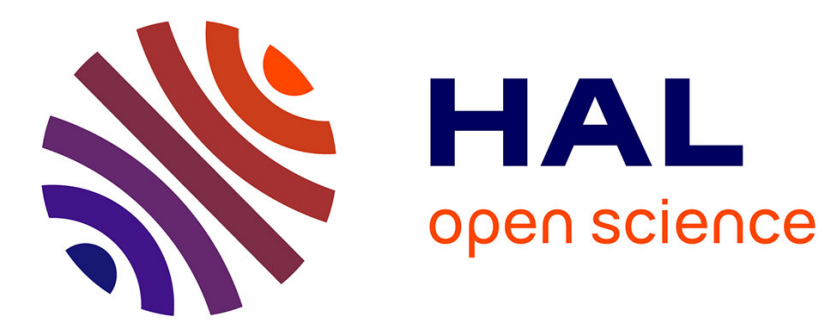

\title{
Optimal control problem in bond graph formalism
}

Omar Mouhib, Audrey Jardin, Wilfrid Marquis-Favre, Eric Bideaux, Daniel

Thomasset

\section{To cite this version:}

Omar Mouhib, Audrey Jardin, Wilfrid Marquis-Favre, Eric Bideaux, Daniel Thomasset. Optimal control problem in bond graph formalism. Simulation Modelling Practice and Theory, 2009, 17 (1), pp.240-256. 10.1016/j.simpat.2008.04.011 . hal-00368302

\section{HAL Id: hal-00368302 https://hal.science/hal-00368302}

Submitted on 28 Mar 2019

HAL is a multi-disciplinary open access archive for the deposit and dissemination of scientific research documents, whether they are published or not. The documents may come from teaching and research institutions in France or abroad, or from public or private research centers.
L'archive ouverte pluridisciplinaire HAL, est destinée au dépôt et à la diffusion de documents scientifiques de niveau recherche, publiés ou non, émanant des établissements d'enseignement et de recherche français ou étrangers, des laboratoires publics ou privés. 


\title{
Optimal control problem in bond graph formalism*
}

\author{
Omar Mouhib Audrey Jardin Wilfrid Marquis-Favre \\ Eric Bideaux Daniel Thomasset \\ Laboratoire AMPÈRE - UMR CNRS 5005 \\ Institut National des Sciences Appliquées de Lyon \\ 25, avenue Jean Capelle, F-69621 Villeurbanne Cedex \\ e-mail: < firstname $>$.<lastname $>$ @insa-lyon.fr
}

\begin{abstract}
This paper presents a new way to derive an optimal control system for a specific optimisation problem, based on bond graph formalism. The procedure proposed concerns the optimal control of linear time invariant MIMO systems and can deal with both cases of the integral performance index, these correspond to dissipative energy minimization and output error minimization. An augmented bond graph model is obtained starting from the bond graph model of the system associated with the optimal control problem. This augmented bond graph, consisting of the original model representation coupled to an optimizing bond graph, supplies, by its bicausal exploitation, the set of differential-algebraic equations that analytically give the solution to the optimal control problem without the need to develop the analytical steps of Pontryagin's method. The proof uses the Pontryagin Maximum Principle applied to the port-Hamiltonian formulation of the system.
\end{abstract}

Keywords: Optimal control, dissipative energy minimization, output error minimization, bond graph, Pontryagin Maximum Principle, port-Hamiltonian system, bicausality.

\section{Introduction}

Bond graph language proves to be a very efficient tool for modelling, analysing and designing mechatronic systems from an energy and dynamic point of view $[4,13]$. The main idea presented in this paper is to introduce an optimal control problem into bond graph formalism. The perspective is to couple this formulation with a sizing methodology of mechatronic systems using bond graph language and the state space inverse model approach. This methodology of sizing, based on dynamic and energy criteria using bond graph language, was developed at the Laboratoire d'Automatique Industrielle ${ }^{1}[5,6,7,8]$. The objective is to transpose the

${ }^{*}$ This work has been carried out within the scope of the RNTL-METISSE project and authorized by the French Ministry of National Education and Research.

${ }^{1}$ Since January 1, 2007, the LAI, the CEGELY and a team of environmental genomic microbial have been regrouped to be called 'Ampère' 
optimization problem into bond graph formalism so that its exploitation will solve this problem. The procedure for building the bond graph representation of the given optimal control problem is presented; this enables the set of differential-algebraic equations to be derived that give the solution to the optimal control problem. In fact the equations obtained are derived graphically by assigning the bicausality to this augmented bond graph representation, avoiding the analysis usually involved by applying the Pontryagin Maximum Principle [14].

This paper is lays out all our research work published up until now $[3,15,17,18]$, moreover this research presents the propositions within a more general framework of linear time invariant MIMO systems. This research was started by some of the authors of this paper and the first results were given at the 7th ICBGM conference in New Orleans [15]. This paper consisted of building an augmented bond graph from the bond graph model of a system, where the assignment of the bicausality gives the solution to the optimal control problem. The method has been developed using the example of a DC motor and the optimization objective taken was the minimization of dissipative energy. The optimisation objective is expressed as the integral of a quadratic form of the state space vector and the control input to be determined. The optimal control problem was formulated analytically and, in parallel, by means of bond graph language. This very simple example has been chosen to understand better the whole bond graph generation mechanism. The steps of bond graph formulation specify a systematic procedure. This procedure aims at automatically generating the corresponding bond graph representation and a proof of its effectiveness. It has been presented in [17]. The class of system that the procedure can deal with was formerly restricted to linear time invariant SISO systems before it was extended to linear time invariant MIMO systems. Recently a new investigation of the bond graph construction of an optimal control problem for another performance index has been carried out. This studys the output trajectory tracking [18]. In this case the performance index may be expressed as a quadratic form of error to be minimized between a specified output and the actual output. The control variable is also taken into account by using a weighting factor. Here the two procedures are grouped into only one procedure. This enables the reader to apply his optimal control problem without returning to the previous references and it takes into account the various types of the input and output (effort or flow).

The key idea of the proof of this procedure is to apply the Pontryagin Maximum Principle to a generic port-Hamiltonian system. Port-Hamiltonian system is an analytical expression of the dynamic equations governing a model that mathematically clearly reflects the energy topology of the system model $[16,19]$. Boundary conditions are supposed fixed, in particular for both final time and final state and, finally, no constraint exists on either inputs or states. This voluntary restricted hypothetical framework has enabled the first step in the coupling of optimisation and bond graph to be clearly investigated and offers encouraging perspectives for future work. A simple numerical method for solving the problem of finding the initial costate conditions from the initial set of boundary conditions has been implemented and is given in [3]. This point will not be detailed in this paper and the reader will be able to consult the reference above for more details. 


\section{Organization of the paper}

The paper is organized as follows. Section 3 synthesizes the procedure of the bond graph construction of an optimal control problem in the form of a proposition where the integral performance index may contain dissipative energy and/or an output error to minimize. This gives the construction steps of this augmented bond graph representation and it also summarizes the conditions of how the procedure can be applied. Its demonstration justifies the former procedure and proves its effectiveness. This is given in section 4. The developments concerning the optimal control problem are based on the Pontryagin Maximum Principle and the proof of the procedure's effectiveness uses the port-Hamiltonian concept.

The proposed graphical procedure is tested on an example of three masses in series shown in section 5. This graphical procedure shows that we can obtain the same result as the one that we would obtain by using classical analytic developments. Section 6 concludes the paper with a summary of the results and suggests future directions of research.

Additionally the two appendices give, respectively, bases on partial dualization in the bond graph used for proof of the effectiveness of the procedure (appendix A) and on the bicausality concept for the bond graph exploitation that provides the optimal control system (appendix B).

\section{Procedure for the construction of a bond graph opti- mal control problem}

This section proposes a systematic procedure for generating the bond graph of the optimal control problem within a general framework of linear time invariant MIMO systems when the optimisation objectives are minimizing the dissipation and the tracking of a reference trajectory. The control vectors to be determined can be effort and/or flow variables, the same is true for the nature of the output vectors for tracking/specifying. Thus this optimal control bond graph representation would be exploitable for directly determining the optimal control solution without developing the analytical steps of Pontryagin's method. However, due to the relative novelty of the approach presented, it is essential to recall its conditions of application:

Let:

- a linear time invariant model of a MIMO system and its bond graph representation,

- the input controls to determine with respect to the integral performance index to minimize. This integral is the terms of half of a control-based quadratic form, a dissipative energy, and/or a quadratic error between specified outputs and the actual outputs.

- fixed-boundary conditions for the time and state space and

- no constraint on inputs or on state.

So, knowing a trajectory defined by $\left\{y_{r}(t)\right\}_{t \in\left[t_{0}, t_{f}\right]}$, where $t_{0}$ and $t_{f}$ indicate the horizon of the fixed state, the problem is to determine the control $u$ for the given initial states $x_{0}$. This control minimizes some dissipative energy (power $P_{\text {diss }}$ ) while keeping the output error $y(t)-y_{r}(t)$ bounded. This problem can be formalized as a problem of quadratic error minimization on the time boundary $\left[t_{0}, t_{f}\right]$ :

$$
V=\int_{\mathrm{t}_{0}}^{\mathrm{t}_{\mathrm{f}}} \frac{1}{2}\left[u^{\mathrm{T}} \cdot R_{u}^{-1} \cdot u+P_{\text {diss }}+\left(y-y_{r}\right)^{\mathrm{T}} \cdot Q \cdot\left(y-y_{r}\right)\right] d t
$$


The following proposition enables the bond graph representation of the given optimal control problem to be obtained.

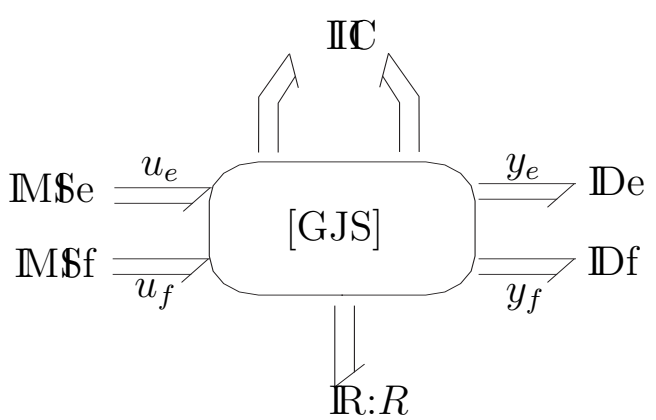

Figure 1: Bond graph model

Proposition : for all optimal control problems of a linear time invariant MIMO system, with the integral performance index of the form (1) and with given boundary conditions; we can build, from the bond graph representation of the system under study (Fig. 1), an augmented bond graph representation (Fig. 2) where its bicausal exploitation enables the system of equations that provide the optimal solution to be derived.

According to figure 1 and figure 2, and in accordance with the integral performance index of the equation (1): $u=\left[\begin{array}{ll}u_{e}^{T} & u_{f}^{T}\end{array}\right]^{T}$ is the vector of the inputs of the system, $y=\left[\begin{array}{ll}y_{e}^{T} & y_{f}^{T}\end{array}\right]^{T}$ is the vector of the outputs, $y_{r}=\left[y_{r_{e}}^{T} y_{r_{f}}^{T}\right]^{T}$ is the reference trajectory, $R_{u}=\left[\begin{array}{ll}R_{u_{e}} & \\ & R_{u_{f}}^{-1}\end{array}\right]$ where $R_{u_{e}}$ and $R_{u_{f}}$ are control weighted matrices and $Q=\left[\begin{array}{cc}Q_{e} & \\ & Q_{f}\end{array}\right]$ where $Q_{e}$ and $Q_{f}$ are weighting matrices assumed diagonal. The subscripts ' $e^{\prime}$ and ' $f$ ' indicate the effort and flow respectively. $\quad P_{\text {diss }}$ is the dissipation power at certain multiport R-elements, $R=\left[\begin{array}{ll}R_{\mathrm{rr}} & R_{\mathrm{rc}} \\ R_{\mathrm{cr}} & R_{\mathrm{cc}}\end{array}\right]$ is the characteristic matrix of the multiport $\mathrm{R}-$ element and $T=\left[\begin{array}{cc}R_{\mathrm{rr}}^{\mathrm{T}} & -R_{\mathrm{cr}}^{\mathrm{T}} \\ -R_{\mathrm{rc}}^{\mathrm{T}} & R_{\mathrm{cc}}^{\mathrm{T}}\end{array}\right]\left(\begin{array}{lll}\mathrm{r}-\mathrm{resp} . & \mathrm{c}-\end{array}\right.$ corresponding to a R-element port in resistance - resp. conductance- causality when the bond graph is in integral causality -see appendix A-). A multibond graph notation [2] has been adopted. In this notation GJS stands for Generalized Junction Structure.

The construction steps of this augmented bond graph representation of the given optimal control problem can be given according to the following procedure:

\section{Procedure:}

1. For each control to be optimally determined, add to the bond graph model an R-element characterized by the factors of the square input term in the performance index. This Relement is connected to a junction inserted into the control source bond and corresponding to the nature of the control variable i.e. a 0(resp. 1)-junction for an effort (resp. flow). The added R-element may find its physical interpretation in some dissipative phenomena of a non-ideal energy supply. 


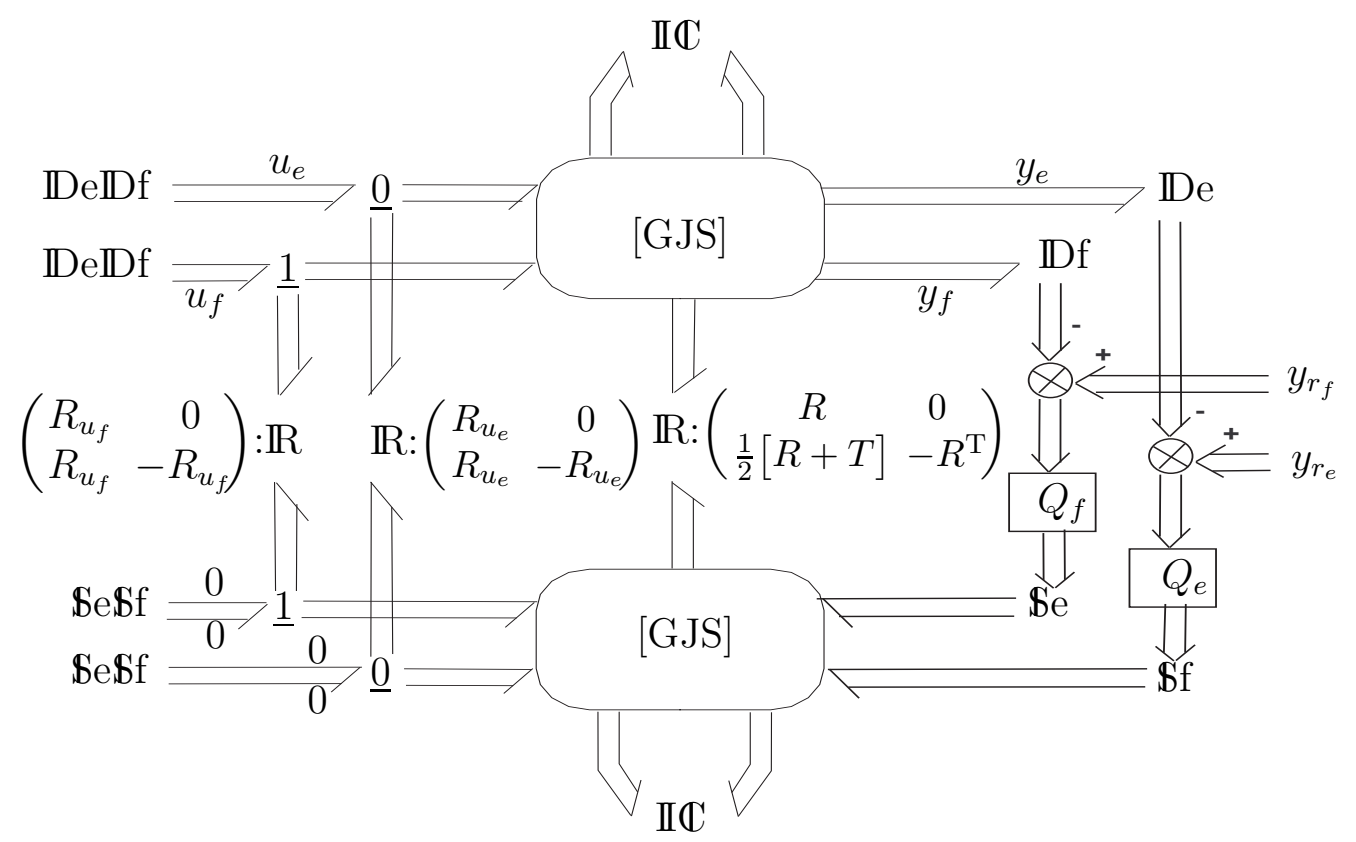

Figure 2: Generic bond graph representation of the optimal control problem

2. Duplicate the bond graph model with its parameters except for the R-elements. For the R-elements corresponding to the model dissipation phenomena, the characteristic matrices are transposed and the sign reversed. For the R-elements added at step 1, the characteristic matrices have their sign reversed. The duplicated representation is hereafter called optimizing bond graph.

3. For the determination of each optimal effort control, couple the corresponding R-elements respectively in the model and the optimizing bond graphs by a multiport $\mathrm{R}$-element with the constitutive matrix (2).

$$
\left(\begin{array}{cc}
R_{u_{e}} & 0 \\
R_{u_{e}} & -R_{u_{e}}
\end{array}\right)
$$

Apply the same operation for each optimal flow control with the matrix (3).

$$
\left(\begin{array}{cc}
R_{u_{f}} & 0 \\
R_{u_{f}} & -R_{u_{f}}
\end{array}\right)
$$

4. For each dissipative phenomenon involved in the given integral performance index, couple the corresponding R-elements respectively in the model and the optimizing bond graphs by a multiport $\mathrm{R}$-element with the constitutive matrix (4) :

$$
\left(\begin{array}{cc}
R & 0 \\
\frac{1}{2}[R+T] & -R^{\mathrm{T}}
\end{array}\right)
$$


5. If there is an output error to minimize in the performance index, replace, in the optimizing bond graph, the effort detectors by flow sources and the flow detectors by effort sources. These sources are the errors between specified outputs and the actual outputs multiplied by the corresponding weight coefficient, i.e. $Q_{e}$ for effort output error and $Q_{f}$ for flow output error.

6. Replace in the bond graph model the source elements involved in the optimal controls by double detectors and mirror them by double sources at the same place on the optimizing bond graph. The double sources impose both null efforts and flows.

7. Assign bicausality to the bond graph obtained. Bicausality is propagated from the double sources to the double detectors and through the R-elements added at step 1 . The analytical exploitation of the bicausal bond graph representation obtained provides the system equations and the optimal control solutions to the given initial problem.

Note that the procedure can deal with problems when it is not necessarily a question of minimizing dissipative energy and a quadratic output error at the same time. In the case where only dissipation is taken into consideration in the performance index, procedure at step 5 will be abandoned. Likewise, for the optimal control problem with only a quadratic output error as a minimization objective, procedure at step 4 will not be taken into account since all R-elements corresponding to dissipative phenomena are left uncoupled. Also when only certain inputs and certain dissipation phenomena are involved in the performance index, the corresponding multiport R-elements are left uncoupled in the step 4 procedure for the uninvolved dissipation phenomena. For the inputs excluded from the optimal control design problem, the multiport source elements are not replaced by double detectors and no R-element is added at the step 1 procedure.

\section{Proof of the effectiveness of the procedure}

This section proves that the bond graph representation obtained by the above procedure corresponds well to the given optimal control problem. The proof is based on the port-Hamiltonian system concept $[16,19]$ that has been proven to be the geometric counterpart of the graphical bond graph representation.

\section{Port-Hamiltonian system}

Consider a physical system with the total stored energy represented by its Hamiltonian $H(x) . H$ is expressed in the linear case as a quadratic form of $x=\left[x_{\mathrm{C}}^{\mathrm{T}} x_{\mathrm{I}}^{\mathrm{T}}\right]^{\mathrm{T}}$, this is the state space vector corresponding to the energy variables of elements $C$ and $I$ respectively (equation $5)$ :

$$
H(x)=\frac{1}{2} x^{\mathrm{T}} \cdot \mathrm{H} \cdot x
$$

where the Hessian matrix $\mathrm{H}$ is symmetric, due to the reversibility property and thus because it verifies the Maxwell reciprocity property [13] and is definite positive.

A generalization of a class of the Hamiltonian systems called the port-Hamiltonian makes it possible to deal with open systems where they can exchange energy with their environment. 
In the hypothetical framework of linear time invariant systems and in the context of this work, the port-hamiltonian systems are given by (6) [19].

$$
\left\{\begin{array}{l}
\dot{x}=\mathrm{J} \cdot \frac{\partial H(x)}{\partial x}+\mathrm{g}_{u} \cdot u+\mathrm{g}_{y} \cdot u_{y} \\
y_{u}=\mathrm{g}_{u}^{\mathrm{T}} \cdot \frac{\partial H(x)}{\partial x} \\
y=\mathrm{g}_{y}^{\mathrm{T}} \cdot \frac{\partial H(x)}{\partial x}
\end{array}\right.
$$

where $u=\left[\begin{array}{ll}u_{e}^{\mathrm{T}} & u_{f}^{\mathrm{T}}\end{array}\right]^{\mathrm{T}}$ is the power variable vector of inputs and $y_{u}$ the vector of their power conjugate variables. $y=\left[\begin{array}{ll}y_{e}^{\mathrm{T}} & y_{f}^{\mathrm{T}}\end{array}\right]^{\mathrm{T}}$ is the power variable vector of outputs and $u_{y}$ the vector of their power conjugate variables. These vectors, at the environmental, ports are defined by the input matrix $g_{u}$ and the output matrix $g_{y}$. Note that $u_{y}=\left[u_{y_{e}}^{\mathrm{T}} u_{y_{f}}^{\mathrm{T}}\right]^{\mathrm{T}}=0$, however we keep track of this term for the bond graph translation of the optimization problem. $J$ is a Poisson Structure matrix satisfying the skew-symmetry property i.e. $\mathrm{J}=-\mathrm{J}^{\mathrm{T}}[16]$.

In the case of systems with energy dissipation, dissipative elements can be connected to some of the ports and the port hamiltonian system expression will be written (7):

$$
\left\{\begin{array}{l}
\dot{x}=\mathrm{J} \cdot \frac{\partial H(x)}{\partial x}+\mathrm{g}_{u} \cdot u+\mathrm{g}_{y} \cdot u_{y}-\mathrm{g}_{R} \cdot u_{R} \\
y_{u}=\mathrm{g}_{u}^{\mathrm{T}} \cdot \frac{\partial H(x)}{\partial x} \\
y=\mathrm{g}_{y}^{\mathrm{T}} \cdot \frac{\partial H(x)}{\partial x} \\
y_{R}=\mathrm{g}_{R}^{\mathrm{T}} \cdot \frac{\partial H(x)}{\partial x}
\end{array}\right.
$$

where $u_{R}$ and $y_{R}$ are the power variable vectors associated with the dissipative phenomena and $g_{R}$ is a matrix associated with the junction structure transformation between the storage elements and the R-elements.

By an appropriate dualization of the dissipative constitutive laws, it is always possible to consider that the inputs are efforts $\left(u_{R}=e_{R}\right)$ while the outputs are flows $\left(y_{R}=f_{R}\right)$. Thus, in the linear case, the dissipative constitutive laws can be written:

$$
e_{\mathrm{R}}=R_{\mathrm{R}} \cdot f_{\mathrm{R}}
$$

where $R_{R}$ is the matrix that characterizes the dissipative phenomena.

A generic causal bond graph representation of equations 7 and 8 is given in figure 3 [13]. For the sake of conciseness, partial dualization has been used for R-element ports with conductance causality [1]. This introduces symplectic gyrators that inverse the effort and flow roles and enable every case of multiport R-elements causality assignment in a unique causal representation to be treated without loss of generality in the demonstration (see appendix A). In figure 3 the symplectic gyrators are encapsulated in the block [GJS]. Also, the vector $f_{R}$ can now be expressed in terms of the state vector:

$$
f_{\mathrm{R}}=\mathrm{g}_{R}^{\mathrm{T}} \cdot \frac{\partial \mathrm{H}(x)}{\partial x}=\mathrm{g}_{\mathrm{R}}^{\mathrm{T}} \cdot \mathrm{H} \cdot x
$$

Substitution of equations 8 and 9 leads to the form of a port-Hamiltonain model for a dissipative linear invariant system:

$$
\left\{\begin{array}{l}
\dot{x}=[\mathrm{J}-\mathrm{S}] \cdot \mathrm{H} \cdot x+\mathrm{g}_{u} \cdot u+\mathrm{g}_{y} \cdot u_{y} \\
y_{u}=\mathrm{g}_{u}^{\mathrm{T}} \cdot \frac{\partial H(x)}{\partial x} \\
y=\mathrm{g}_{y}^{\mathrm{T}} \cdot \mathrm{H} \cdot x
\end{array}\right.
$$




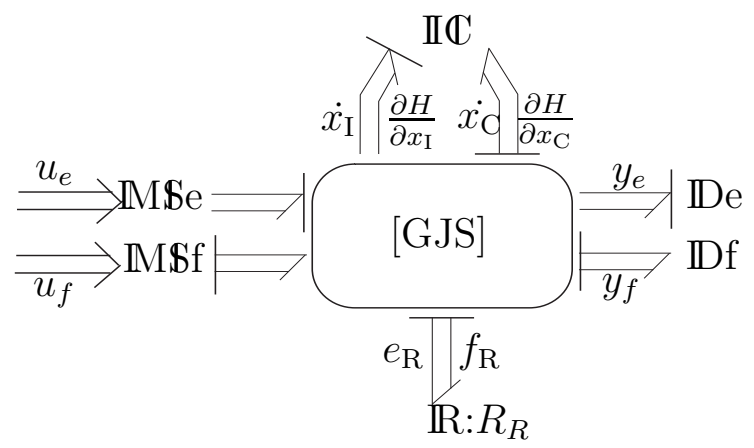

Figure 3: Generic causal bond graph representation for a port-Hamiltonian system

Where $\mathrm{S}$ is a matrix defined by equation (11)

$$
\mathrm{S}=\mathrm{g}_{R} \cdot R_{R} \cdot \mathrm{g}_{R}^{\mathrm{T}}
$$

Note that the matrix $R_{R}$, which characterizes the multiport R-element in the figure 3 , differs from the matrix of figure 1 due to dualization.

\section{Application of the Pontryagin Maximum Principle to the port-Hamiltonian sys- tem}

We consider the integral performance index of the form (1). It can be written:

$$
V=\int_{\mathrm{t}_{0}}^{\mathrm{t}_{\mathrm{f}}} \frac{1}{2}\left[u^{\mathrm{T}} \cdot \mathrm{R}_{u}^{-1} \cdot u+e_{R}^{\mathrm{T}} \cdot f_{R}+\left(y-y_{r}\right)^{\mathrm{T}} \cdot Q \cdot\left(y-y_{r}\right)\right] d t
$$

where the dissipation power $P_{\text {diss }}$ has been expressed as the inner product of the power conjugate vectors of the $\mathrm{R}$-elements $\left(P_{\text {diss }}=e_{\mathrm{R}}^{\mathrm{T}} \cdot f_{\mathrm{R}}\right)$. The coefficient $\frac{1}{2}$, without changing the optimisation problem, enables a coefficient 2 in some of the following terms to be eliminated.

The matrix $R_{u}$ may be interpreted as characterizing some dissipative phenomena embedded in energy supplies thus considered as non-ideal [14]. We implement an extra multiport Relement that does not change the port-hamiltonian model on its own but takes into account the corresponding performance index term in the figure 4 bond graph representation. The application of Pontryagin's Maximum Principle [14] on the cost function (12) and the dynamic constraints (10) leads to the equation system (13):

$$
\left\{\begin{array}{l}
\dot{p}=\frac{\partial H_{p}(p, \lambda, u)}{\partial \lambda} \\
\dot{\lambda}=-\frac{\partial H_{p}(p, \lambda, u)}{\partial p} \\
\frac{\partial H_{p}(p, \lambda, u)}{\partial u}=0
\end{array}\right.
$$

where $\lambda$ is the vector of co-state variables (or covariant vector), usually called Lagrange multipliers of the associated constrained variational problem. $H_{p}$ is a Pontryagin function formed by the integral performance index (12) and port-Hamiltonian system (10). $H_{p}$ is given by:

$$
H_{\mathrm{p}}=\frac{1}{2} u^{\mathrm{T}} \cdot R_{u}^{-1} \cdot u+\frac{1}{2} f_{R}^{\mathrm{T}} \cdot R_{R}^{\mathrm{T}} \cdot f_{R}+\frac{1}{2}\left(y-y_{r}\right)^{\mathrm{T}} \cdot Q \cdot\left(y-y_{r}\right)+\lambda^{\mathrm{T}} \cdot\left[[\mathrm{J}-\mathrm{S}] \cdot \mathrm{H} \cdot x+\mathrm{g}_{u} \cdot u+\mathrm{g}_{y} \cdot u_{y}\right]
$$




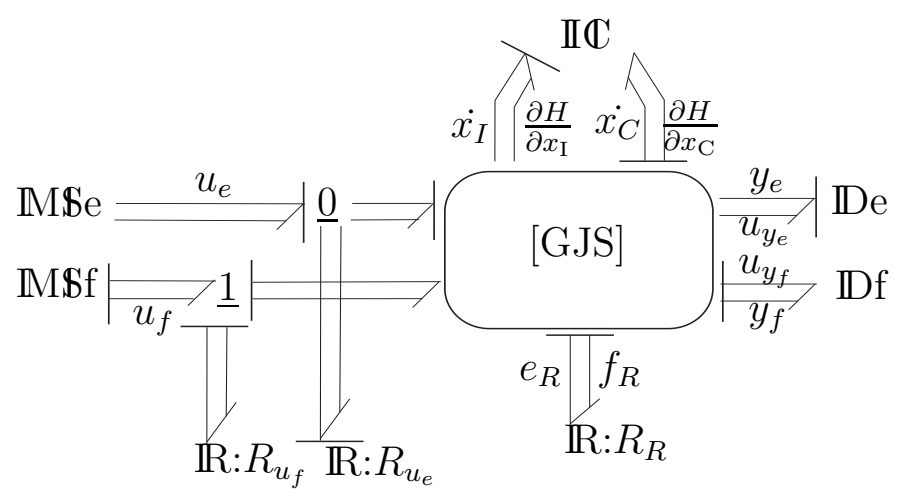

Figure 4: Generic causal bond graph representation for a port-Hamiltonian system with nonideal energy supplies

The equation system (13) together with the boundary conditions $x_{0}=x\left(t_{0}\right)$ and $x_{f}=x\left(t_{f}\right)$ provide the optimal state solution to the optimal control problem. The development of the equation system (13) gives:

$$
\begin{aligned}
& \dot{x}=[\mathrm{J}-\mathrm{S}] \cdot \mathrm{H} \cdot x+\mathrm{g}_{u} \cdot u+\mathrm{g}_{y} \cdot u_{y} \\
& \dot{\lambda}=-\frac{\partial}{\partial x}\left[\frac{1}{2} f_{R}^{\mathrm{T}} \cdot R_{R} \cdot f_{R}+\frac{1}{2}\left(y-y_{r}\right)^{\mathrm{T}} \cdot Q \cdot\left(y-y_{r}\right)\right]-\left[\lambda^{\mathrm{T}} \cdot[\mathrm{J}-\mathrm{S}] \cdot \mathrm{H}\right]^{\mathrm{T}} \\
& R_{u}^{-1} \cdot u+\left[\lambda^{\mathrm{T}} \cdot \mathrm{g}_{u}\right]^{\mathrm{T}}=0
\end{aligned}
$$

Equation (15) can be derived from the figure 4 bond graph representation. In fact the key issue to the bond graph formulation of the optimal control problem resides in the translation of equations (16) and (17) into this language.

Let us first concentrate on equation (16). It can be re-written using the symmetry and skew-symmetry properties of matrices $\mathrm{H}$ and $\mathrm{J}$ respectively, also using equation (9), and the expression (11) of matrix $\mathrm{S}$ as the equation (18) shows.

$$
\dot{\lambda}=-\mathrm{H} \cdot \mathrm{g}_{R} \cdot \frac{1}{2}\left[R_{R}+R_{R}^{\mathrm{T}}\right] \cdot f_{R}-\mathrm{H} \cdot \mathrm{g}_{y} \cdot Q \cdot\left(y-y_{r}\right)+\mathrm{H} \cdot\left[\mathrm{J}+\mathrm{g}_{R} \cdot R_{R}^{\mathrm{T}} \cdot \mathrm{g}_{R}^{\mathrm{T}}\right] \cdot \lambda
$$

Before introducing the bond graph translation of this equation, variable mapping $x_{\lambda}=\mathrm{H}^{-1} \cdot \lambda$ is carried out ( $\mathrm{H}$ is definite positive by hypothesis and thus invertible). This gives :

$$
\begin{aligned}
\dot{x}_{\lambda} & =-\mathrm{g}_{R} \cdot \frac{1}{2}\left[R_{R}+R_{R}^{\mathrm{T}}\right] \cdot f_{R}-\mathrm{g}_{y} \cdot Q \cdot\left(y-y_{r}\right)+\left[\mathrm{J}+\mathrm{g}_{R} \cdot R_{R}^{\mathrm{T}} \cdot \mathrm{g}_{R}^{\mathrm{T}}\right] \cdot \mathrm{H} \cdot x_{\lambda} \\
& =\Lambda_{x}+\Lambda_{\lambda}
\end{aligned}
$$

with $\Lambda_{x}=-\mathrm{g}_{R} \cdot \frac{1}{2}\left[R_{R}+R_{R}^{\mathrm{T}}\right] \cdot f_{\mathrm{R}}, \Lambda_{\lambda}=\left[\mathrm{J}+\mathrm{g}_{R} \cdot R_{R}^{\mathrm{T}} \cdot \mathrm{g}_{R}^{\mathrm{T}}\right] \cdot \mathrm{H} \cdot x_{\lambda}+\mathrm{g}_{y} \cdot u_{\lambda}$ and $u_{\lambda}=Q \cdot\left(y_{r}-y\right)$.

The reason for this variable mapping is that the co-state vector $\lambda$ is analog to a co-energy vector in bond graph language while the vector $x_{\lambda}$ is analog to an energy vector. Since this new vector is not the co-state vector and to distinguish it from the state vector $x$, it is called optimizing-state vector. 
A first inspection shows clearly that the second term $\Lambda_{\lambda}$ of the equation (19) second member corresponds to the eigen dynamics of the optimizing-state while the first term $\Lambda_{x}$ is related to the coupling between both the state and optimizing-state systems. Concerning the term $\Lambda_{\lambda}$, it is not difficult to see that it is closely analog to the expression of the state equations (15) as the equation (20) shows.

$$
\dot{x}=\left[\mathrm{J}-\mathrm{g}_{R} \cdot R_{R} \cdot \mathrm{g}_{R}^{\mathrm{T}}\right] \cdot \mathrm{H}+\mathrm{g}_{u} \cdot u+\mathrm{g}_{y} \cdot u_{y} \longrightarrow \Lambda_{\lambda}=\left[\mathrm{J}-\mathrm{g}_{R} \cdot\left(-R_{R}^{\mathrm{T}}\right) \cdot \mathrm{g}_{R}^{\mathrm{T}}\right] \cdot \mathrm{H}+\mathrm{g}_{y} \cdot u_{\lambda}
$$

This can be written:

$$
\begin{array}{r}
\dot{x}=\left[\mathrm{J}-\mathrm{g}_{R} \cdot R_{R} \cdot \mathrm{g}_{R}^{\mathrm{T}}\right] \cdot \mathrm{H}+\mathrm{g}_{u_{e}} \cdot u_{e}+\mathrm{g}_{u_{f}} \cdot u_{f}+\mathrm{g}_{y_{e}} \cdot u_{y_{e}}+\mathrm{g}_{y_{f}} \cdot u_{y_{f}} \\
\longrightarrow \Lambda_{\lambda}=\left[\mathrm{J}-\mathrm{g}_{R} \cdot\left(-R_{R}^{\mathrm{T}}\right) \cdot \mathrm{g}_{R}^{\mathrm{T}}\right] \cdot \mathrm{H}+\mathrm{g}_{y_{e}} \cdot u_{\lambda_{e}}+\mathrm{g}_{y_{f}} \cdot u_{\lambda_{f}}
\end{array}
$$

where $u_{y_{e}}$ and $u_{y_{f}}$ are the conjugate variables of the power variables $y_{e}$ and $y_{f}$ at the effort detector port and flow detector port respectively (Fig. 4). These variables can be considered as two fictitious inputs in the port-Hamiltonian system since they are null. The addition of these inputs in equation (6) allows us to make a comparison by analogy between equation (21) and equation (22). So, by identifying the power variables $u_{\lambda_{e}}=Q_{e} \cdot\left(y_{r_{e}}-y_{e}\right)$ and $u_{\lambda_{f}}=$ $Q_{f} \cdot\left(y_{r_{f}}-y_{f}\right)$ take the same nature as those of the power variables $u_{y_{e}}$ and $u_{y_{f}}$ respectively.

Consequently the figure 3 bond graph partial representation embedding the set $\{\mathbb{I I}, \mathbb{R},[\mathrm{GJS}]\}$ can be reproduced to represent the term $\Lambda_{\lambda}$ contributing to $\dot{\boldsymbol{x}}_{\lambda}$ (Fig. 5 ) just by changing $R_{R}$ into $-R_{R}^{T}$. An Inspection of the term $\Lambda_{x}$ in the equation (19) shows that its contribution stems from

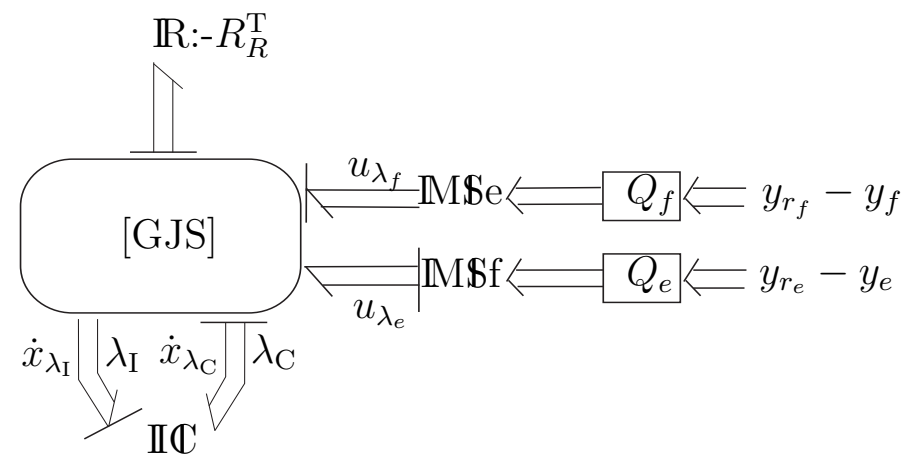

Figure 5: Bond graph translation of the term $\boldsymbol{\Lambda}_{\lambda}$ contribution in equation (19)

the previously introduced multiport R-element through the junction structure transformation characterized by $\mathrm{g}_{R}$. So the term $\frac{1}{2}\left[R_{R}+R_{R}^{\mathrm{T}}\right] f_{R}$ is calculated from the vector $f_{R}$ and contributes to the effort vector of the same multiport R-element. This results in the figure 6 bond graph translation that is the addition of the Fig. 4 and 5 bond graphs where the multiport R-elements have been replaced by a global multiport R-element characterized by the two matrices $R_{R}$ and $-R_{R}^{\mathrm{T}}$ arranged in block diagonal and by the complementary lower extra diagonal submatrix $\frac{1}{2}\left[R_{R}+R_{R}^{T}\right]$. This multiport R-element represents the coupling between the state and the optimizing-state equations. It has been proposed to call the added bond graph representation the optimizing-bond graph, mirroring to some extent the initial one. It is now necessary to treat 


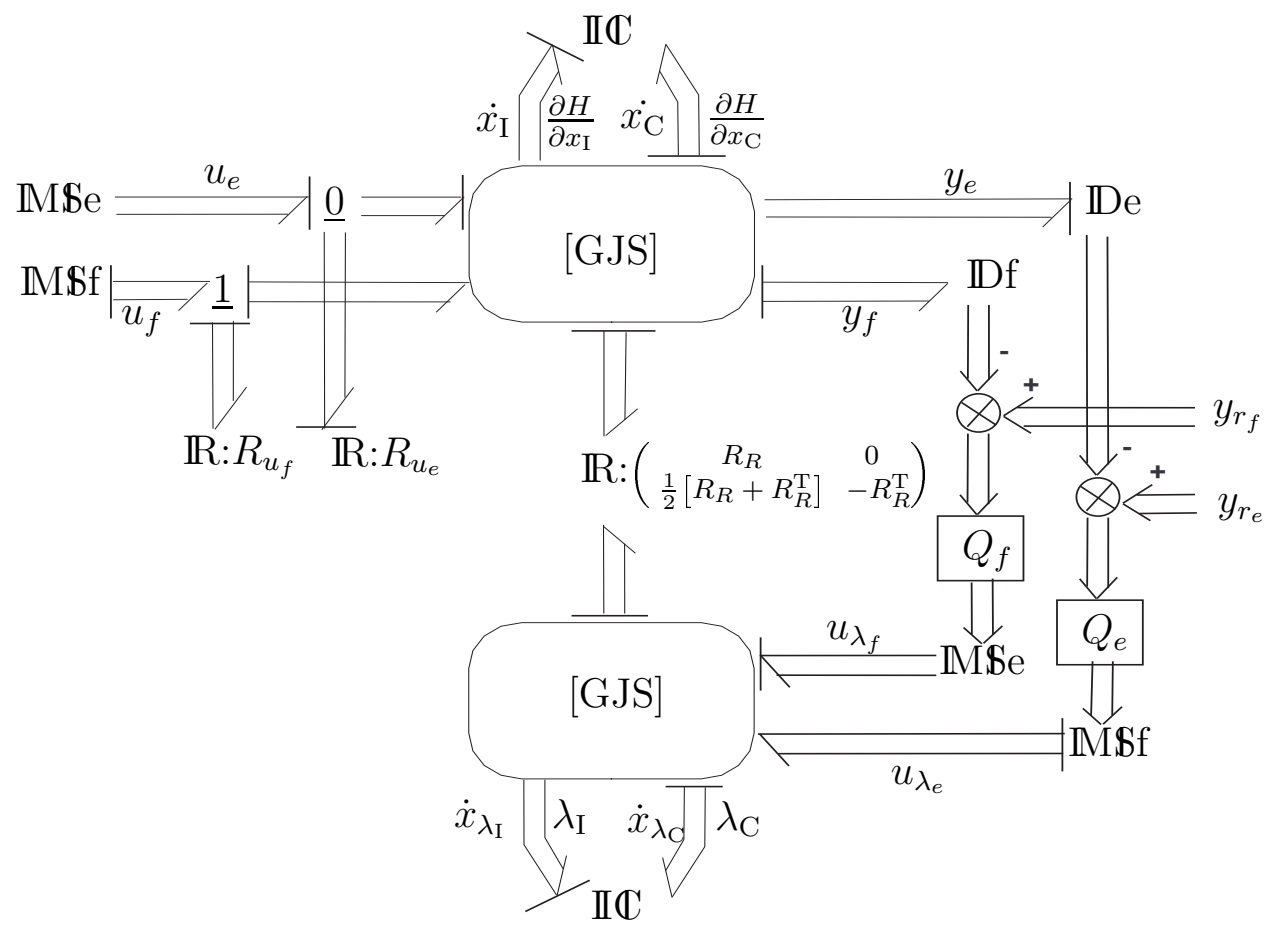

Figure 6: Bond graph translation of both state and optimizing-state equations (15) and (19)

equation (17) which corresponds to the Euler equation with respect to the control vector $u$. First it is re-written as follows:

$$
\begin{aligned}
& R_{u_{e}}^{-1} \cdot u_{e}+\mathrm{g}_{u_{e}}^{\mathrm{T}} \cdot \lambda=0 \\
& R_{u_{f}} \cdot u_{f}+\mathrm{g}_{u_{f}}^{\mathrm{T}} \cdot \lambda=0
\end{aligned}
$$

The equation 23 (24 resp.) can be interpreted as a flow (effort resp.) vector balance between a vector stemming from the control vector $u_{e}\left(u_{f}\right.$ resp.) in the original system and a vector coming from the vector $\lambda$ through the junction structure characterized by $g_{u_{e}}\left(g_{u_{f}}\right.$ resp.). This balance is translated by mirroring, in the optimizing bond graph, the bond graph model between the junction structure and the effort source (figure 7 (a)) (flow source resp. (figure 7 (b))) in the left-hand side part of figure 4 . Likewise, the multiport R-elements are regrouped into a global multiport R-element characterized by the matrix (25) for effort control inputs and by the matrix (26) for flow control inputs.

$$
\begin{aligned}
& \left(\begin{array}{cc}
R_{u_{e}} & 0 \\
R_{u_{e}} & -R_{u_{e}}
\end{array}\right) \\
& \left(\begin{array}{cc}
R_{u_{f}} & 0 \\
R_{u_{f}} & -R_{u_{f}}
\end{array}\right)
\end{aligned}
$$

Now by imposing simultaneously the balance of the two flow vectors and a null effort vector to the optimizing bond graph 0-junction array (figure 7 (a)) and 1-junction array (figure 7 (b)), 
the Euler equations 23 and 24 with respect to the $u_{e}$ and $u_{f}$ are verified. This can be proved by (using the figure 7-(a) vector notations):

$$
\begin{array}{ll}
e_{\mathrm{opt}}=R_{u_{e}} \cdot f-R_{u_{e}} \cdot f_{\mathrm{opt}}=0 & \text { from the second vector characteristic of the R-element } \\
f_{\mathrm{opt}}=-\mathrm{g}_{u_{e}}^{\mathrm{T}} \cdot \lambda & \text { from the flow vector balance } \\
f=R_{u_{e}}^{-1} \cdot e=R_{u_{e}}^{-1} \cdot u_{e} & \text { from the first vector characteristic of the R-element } \\
\text { so } & \\
R_{u_{e}}^{-1} \cdot u_{e}+\mathrm{g}_{u_{e}}^{\mathrm{T}} \cdot \boldsymbol{\lambda}=\mathbf{0} &
\end{array}
$$

and it can also be proved by (using the figure 7-(b) vector notations):

$$
\begin{array}{ll}
e_{\mathrm{opt}}=R_{u_{f}} \cdot f-R_{u_{f}} \cdot f_{\mathrm{opt}}=R_{u_{f}} \cdot f & \text { from the second vector characteristic of the R-element } \\
e_{\mathrm{opt}}=-\mathrm{g}_{u_{f}}^{\mathrm{T}} \cdot \lambda & \text { from the effort vector balance } \\
e=R_{u_{f}} \cdot f=R_{u_{f}} \cdot u_{f}=e_{\mathrm{opt}} & \text { from the first vector characteristic of the R-element } \\
\text { so } & \\
R_{u_{f}} \cdot u_{f}+\mathrm{g}_{u_{f}}^{\mathrm{T}} \cdot \lambda=0 &
\end{array}
$$

It is justified here not to have calculated a priori the optimal controls from the Euler equations

(a)

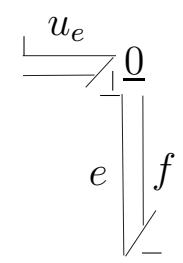

$$
\frac{\mathbb{R}:\left(\begin{array}{cc}
R_{u_{e}} & 0 \\
R_{u_{e}} & -R_{u_{e}}
\end{array}\right)}{\frac{0}{e_{\mathrm{oppt}}}}
$$
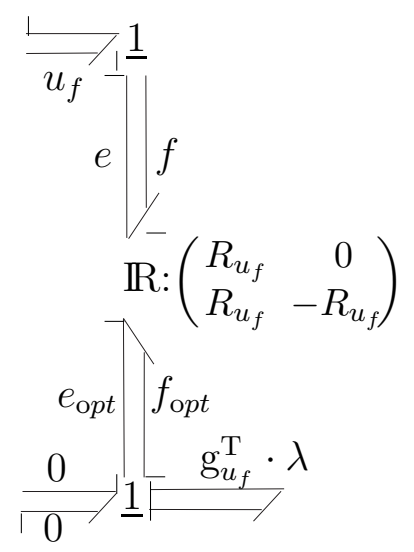

Figure 7: Bond graph translation of Euler equation (24) with respect to $u_{e}$ (a) and $u_{f}$ (b)

in terms of the vector $u$ components.

Finally, the bond graph element that enables both a null effort (resp. flow) vector and a balance of the two flow (resp. effort) vectors to be imposed onto a 0-junction (resp 1-junction) array is a multiport double source null effort vector and null flow vector. It is connected to the 0 -junction (resp 1-junction) array of the figure 7-a (resp 7-b) bond graph. This type of element initializes bicausality $[9,10]$ propagation in the bond graph and thus also requires the presence of a multiport double detector $[6,7,8]$. In the mathematical formulation of the optimal control design problem, the role of the control vector $u$ is changed into an output vector while the power conjugate vector $y$ keeps its original output role. Thus the multiport double detector replaces the original multiport MSe and MSf in the figure 4 bond graph. The final generic causal bond graph representation, in the partial dualization context of the R-elements of the given optimal control problem, is shown in figure 8 and proves, by applying reverse dualization (see appendix A), the proposition and so the effectiveness of the section 3 procedure. 
At this point it is worthwhile noting that, in the application of the procedure, there is no need to transform the original bond graph model into a partially dualized version. Dualization has been used in this section only for a concise demonstration and the procedure applies to a traditional bond graph representation displaying every element of the set $\{$ Se, Sf, I, C, R, TF, GY, 1, 0\}. The correspondence between the different multiport R-element matrices of the figure 8 generic bond graph and that of figure 2 is developed in appendix A.

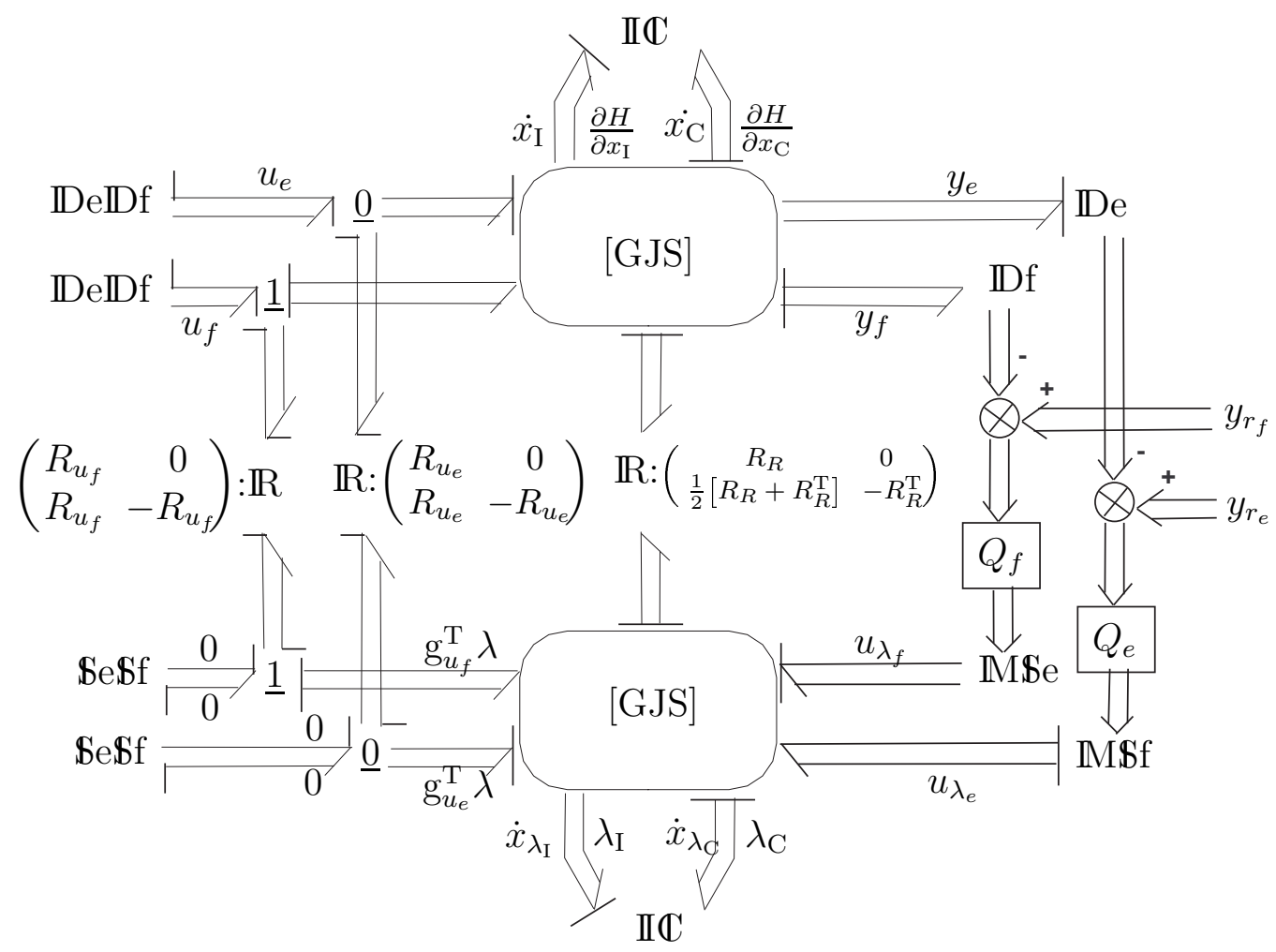

Figure 8: Generic causal bond graph representation of an optimal control problem

\section{Illustrative example: three masses in series}

\subsection{Bond graph formulation}

This example illustrates a case where both effort and flow output errors, energy dissipation and input minimization are included in the integral performance index. The three masses in series model is presented in figure 9 . It consists of three masses in translation, joined by springs and dampers in parallel. The masses are characterised by mass parameters $m_{i}, i=1,2,3$, springs by compliance coefficient $\frac{1}{k_{i}}$ and dampers by a viscous friction parameter $b_{i}$. We suppose that there is no friction between the masses and the ground. An effort input $F(t)$ is imposed on the first mass.

The model is linear and, In the optimal control context, we aim at determining the effort $F$ that minimizes simultaneously some dissipation due to the first damper and the quadratic 
error between an output vector $y$ and a vector of desired outputs $y_{r}$. In this problem the initial conditions at $t_{0}$ and the final conditions at $t_{f}$ are given. Let the following integral performance index be:

$$
V=\int_{\mathrm{t}_{0}}^{\mathrm{t}_{\mathrm{f}}} \frac{1}{2}\left(\frac{F^{2}}{R_{F}}+P_{\mathrm{b}_{1}}+\left(y-y_{r}\right)^{2}\right) d t
$$

where $R_{F}$ is a control weighted factor and $P_{\mathrm{b}_{1}}$ is the dissipation power in the first damper. For this example, the weighting matrix $Q$ is taken as an identity matrix. A specified output vector $y_{r}$ composed of a specified acceleration for the third mass $y_{r_{e}}$ (it might appear as a requirement on an effort output) and of a desired speed for the second mass $y_{r_{f}}$ (i.e. a flow output requirement).

The bond graph representation of this three mass model is given in Fig. 10. It shows the MSe element for the effort source, three I-elements for the three storage phenomena associated with the kinetic energies of the three masses, three C-elements for the energy storage phenomena associated with the stiffness of each spring and three R-elements for the dissipation phenomena associated with each damper.

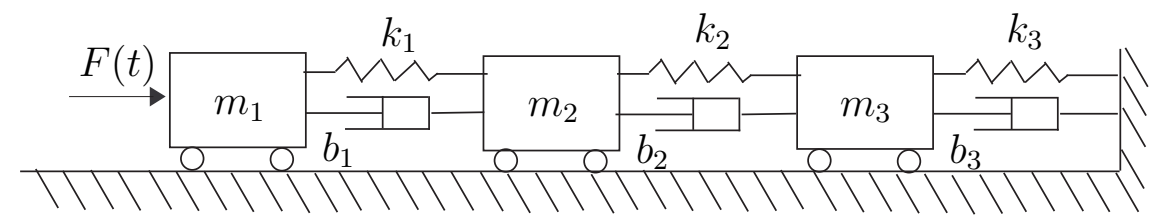

Figure 9: Model of three masses in series

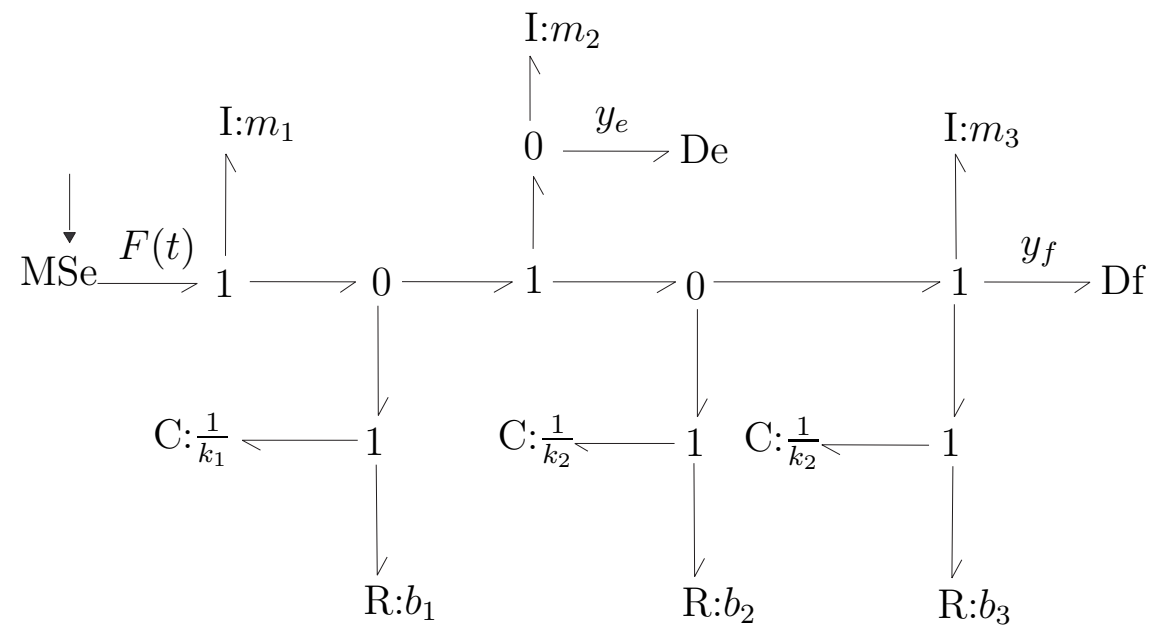

Figure 10: Three masses in series bond graph representation 
The application of the section 3 procedure provides the figure 11 bond graph representation. The bicausality assignment, displayed in this figure, enables the optimal control system (28) to be obtained. This constitutes the final step of the procedure.

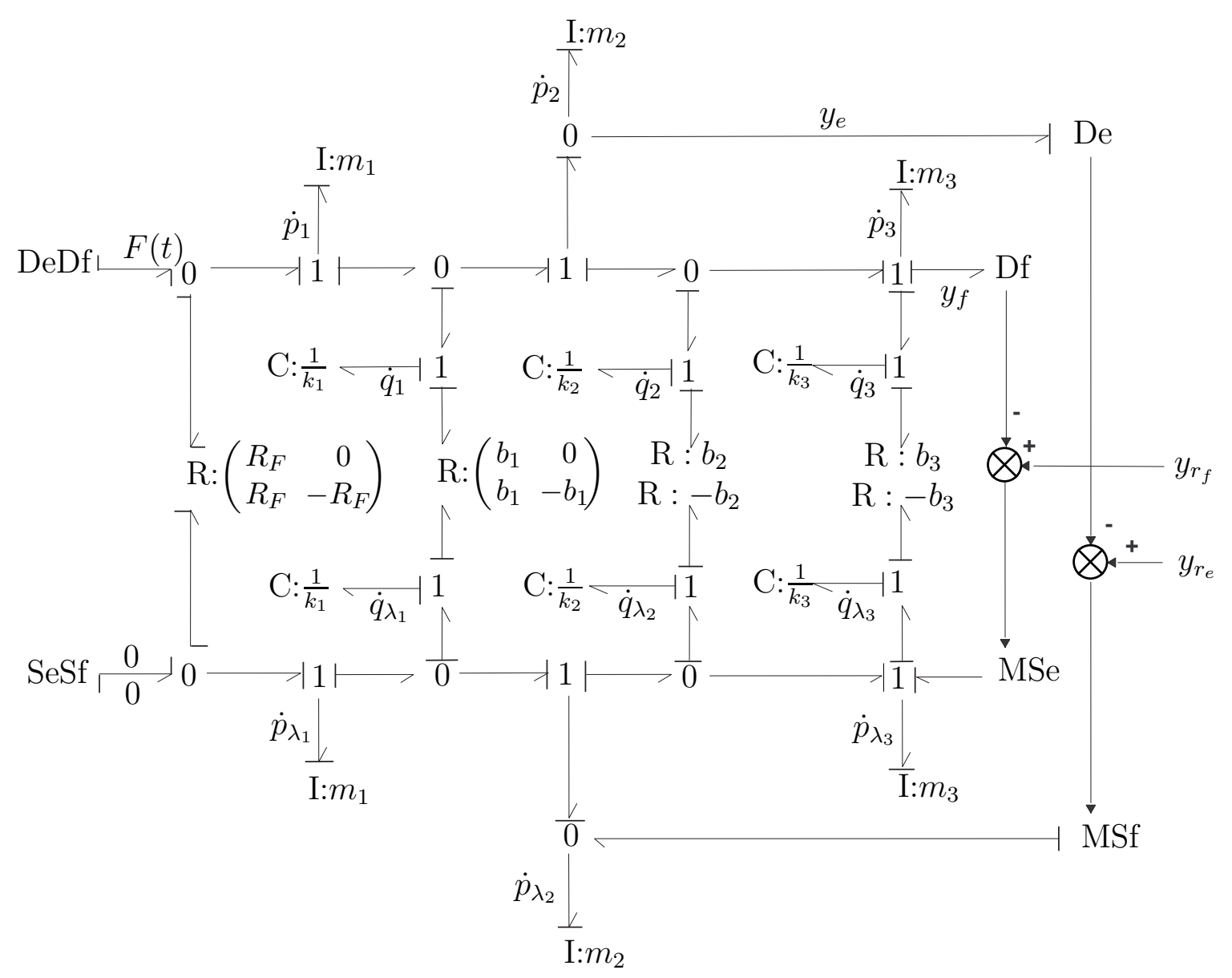

Figure 11: Bond graph representation of the three masses in series optimal control problem 


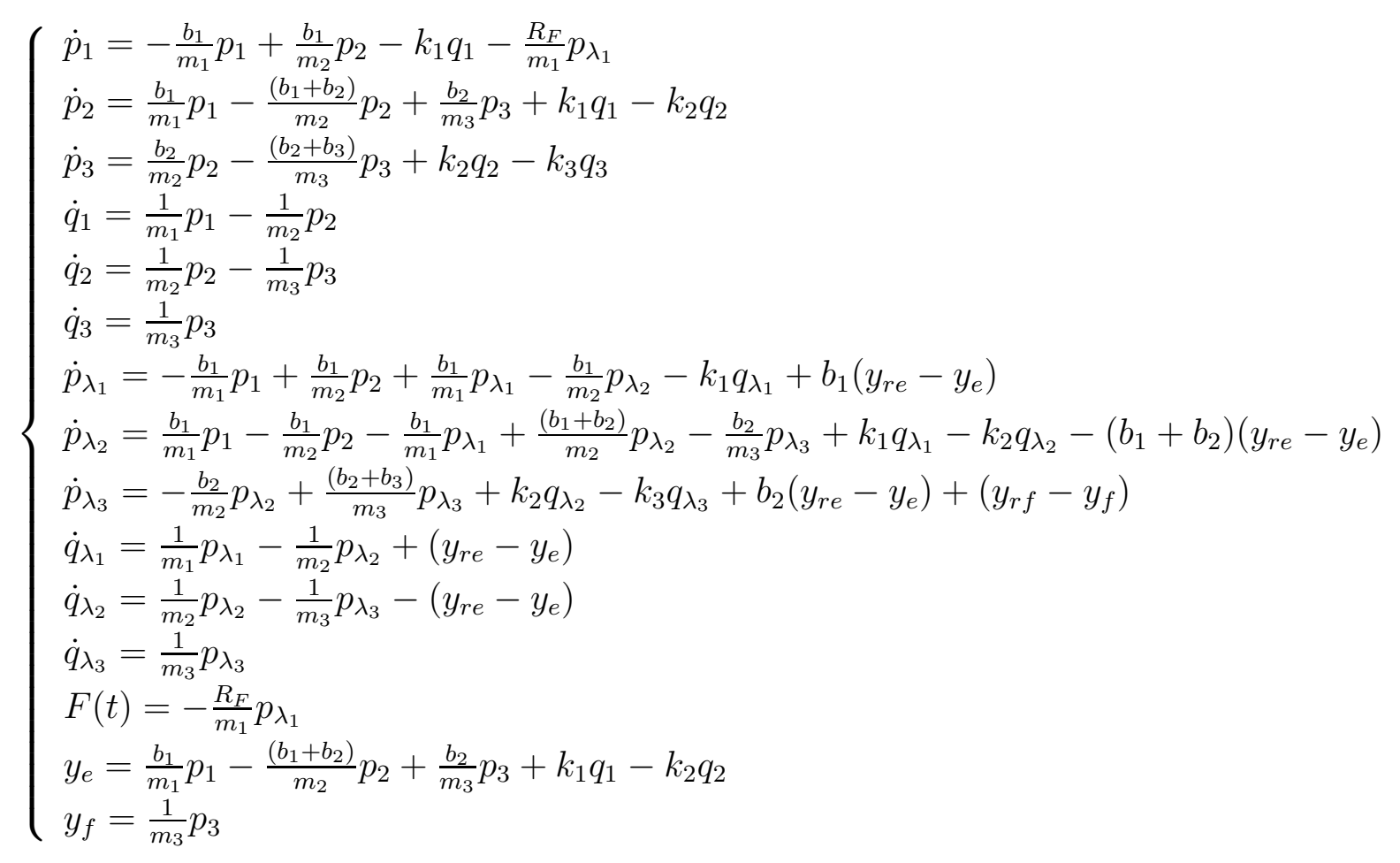

The application of the Pontryagin Maximum Principle leads to the same result but with analytical developments compared to the bond graph graphical approach for deriving the equations.

\subsection{Simulation results}

The key issue for the simulation of the optimal control problem as formulated in this paper is that it requires solving a two point boundary-value problem. In fact the integration of the corresponding system of equations starts from partially known initial conditions (for the state vector) and must reach partially specified final conditions (still for the state vector). The optimizing-state vector is unknown at $t_{0}$ and at $t_{f}$. For linear time invariant (LTI) models, as dealt with in this paper, it will be shown that the initial conditions on this optimizing-state vector can be a priori calculated [21, 22, 23].

For LTI models the solution to the system (29) is given by (30). Here the vector $u(t)$ consists of the components of the vector $y_{r}(t)$ of the specified trajectories.

$$
\begin{aligned}
& \left(\begin{array}{c}
\dot{x}(t) \\
\dot{x_{\lambda}}(t)
\end{array}\right)=A\left(\begin{array}{c}
x(t) \\
x_{\lambda}(t)
\end{array}\right)+B u(t) \\
& \left(\begin{array}{c}
x(t) \\
x_{\lambda}(t)
\end{array}\right)=\exp ^{A\left(t-t_{0}\right)}\left(\begin{array}{c}
x\left(t_{0}\right) \\
x_{\lambda}\left(t_{0}\right)
\end{array}\right)+\int_{t_{0}}^{t} \exp ^{A\left(\tau-t_{0}\right)} B u(\tau) \mathrm{d} \tau
\end{aligned}
$$

The upper half part of equation (30) is used for expressing the final state vector as equation (31) shows. In this expression the matrices $M_{1}$ and $M_{2}$ are determined by simulating the bond graph model of the optimization problem between $t_{0}$ and $t_{f}$, with the specified trajectories set 
to 0 and with a series of arbitrary initial conditions for the state and optimizing-state vectors. For convenience reasons, every initial condition is set to 0 except one which is set to 1 . The final state is then calculated and determines one column of $\left[M_{1} M_{2}\right]$. By repeating this for each state and each optimizing-state initial condition determines every column of $\left[\begin{array}{ll}M_{1} & M_{2}\end{array}\right]$.

$$
x\left(t_{f}\right)=\left[\begin{array}{ll}
M_{1} & M_{2}
\end{array}\right]\left(\begin{array}{c}
x\left(t_{0}\right) \\
x_{\lambda}\left(t_{0}\right)
\end{array}\right)+N_{1}
$$

$N_{1}$ is obtained by simulating twice the bond graph model of the optimization problem with the same initial conditions. The first simulation is carried out with the specified trajectories and the second one without them. The difference between the final state vectors thus calculated determines $N_{1}$.

This approach is preferred to the one consisting of calculating an exponential of matrix because of the recognized difficulties of this calculus [24], in particular for the second term of the solution in equation (30). The key point with this approach is to always keep the specified time horizon $\left[t_{0}, t_{f}\right]$ in the simulations.

Then introducing the specified initial and final conditions for the state vector enables the initial optimizing-state vector to be a priori determined by equation (32).

$$
x_{\lambda_{0}}=M_{2}^{-1}\left[x_{f}-M_{1} x_{0}-N_{1}\right]
$$

The approach described above has been applied to the example of the three masses (Fig. 9). Table 1 lists the parameters used in the model and the optimization problem. The calculated initial optimizing-state vector is:

$$
\left\{\begin{array}{l}
p_{\lambda 1_{0}}=6.999 \\
p_{\lambda 2_{0}}=-18.582 \\
p_{\lambda 3_{0}}=28.908 \\
q_{\lambda 1_{0}}=3.339 \\
q_{\lambda 2_{0}}=-3.134 \\
q_{\lambda 3_{0}}=3.325
\end{array}\right.
$$

The simulations of the optimal problem for the example of the three masses have been carried out using MS1 software [25]. Figures 12 to 15 show the results for the state variables, the optimal control $F_{\text {opt }}(t)$, the outputs with their specified trajectories, and the dissipated energy in the dampers respectively.

\section{Conclusion}

In this paper a procedure and the proof of its effectiveness for representing the optimal control problems in the bond graph language has been given. The type of optimal control problem presented concerns linear time invariant MIMO systems where the integral performance index corresponded to the dissipative energy minimization and the output error to minimize. Starting from the bond graph of the model, the procedure enables the formulation to be set up exclusively at a graphical (namely bond graph) level. The proof uses the Pontryagin principle applied to the port-Hamiltonian formulation of the system.

The example of the three masses in series presented in section 6 illustrated a case where not all the dissipation phenomena are considered in the integral performance index. This proves 


\begin{tabular}{|l|}
\hline Model parameters \\
\hline$m_{1}=m_{2}=m_{3}=5.0 \mathrm{~kg}$ \\
$k_{1}=k_{2}=k_{3}=2.0 \mathrm{~N} / \mathrm{m}$ \\
$b_{1}=b_{2}=b_{3}=0.01 \mathrm{~N} /\left(\mathrm{ms}^{-1}\right)$ \\
\hline Optimization problem parameters \\
\hline$t_{0}=0 \mathrm{~s}$ \\
$t_{f}=50 \mathrm{~s}$ \\
$R_{F}=1.0$ \\
$y_{r_{e}}(t)=\cos (0.3 t)$ \\
$y_{r_{f}}(t)=\cos (t)$ \\
\hline State initial conditions \\
\hline$p_{1_{0}}=0.5 \mathrm{kgms}^{-1}$ \\
$p_{2_{0}}=0.5 \mathrm{kgms}^{-1}$ \\
$p_{3_{0}}=5 \mathrm{kgms}{ }^{-1}$ \\
$q_{1_{0}}=0 \mathrm{~m}$ \\
$q_{2_{0}}=-0.5 \mathrm{~m}$ \\
$q_{3_{0}}=0 \mathrm{~m}$ \\
\hline State final conditions \\
\hline$p_{1_{f}}=0 \mathrm{kgms}$ \\
$p_{2_{f}}=0 \mathrm{kgms}$ \\
$p_{3_{f}}=1.418 \mathrm{kgms}^{-1}$ \\
$q_{1_{f}}=0 \mathrm{~m}$ \\
$q_{2_{f}}=0.381 \mathrm{~m}^{-1}$ \\
$q_{3_{f}}=0 \mathrm{~m}$ \\
\hline
\end{tabular}

Table 1: Parameters for the example of the three masses. 


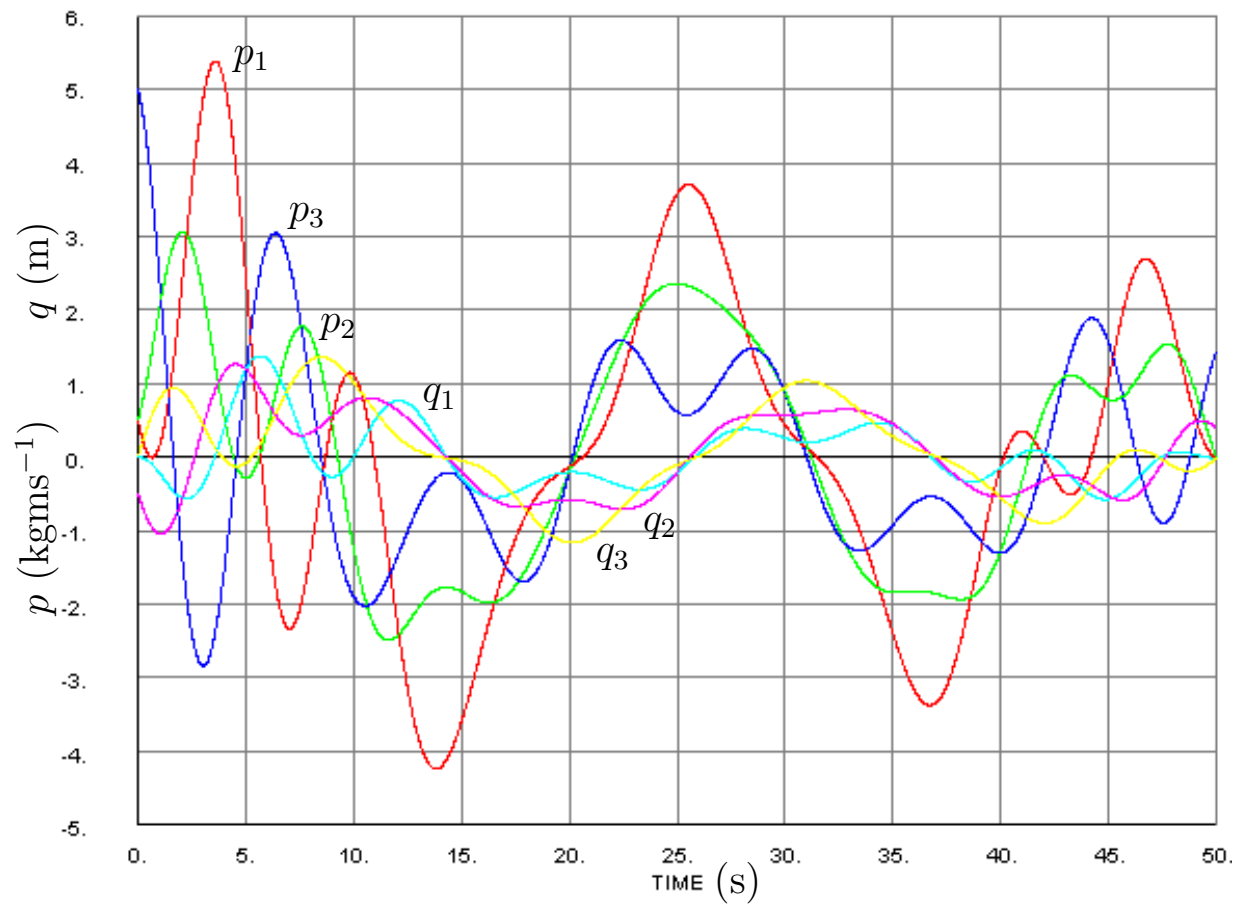

Figure 12: State variables

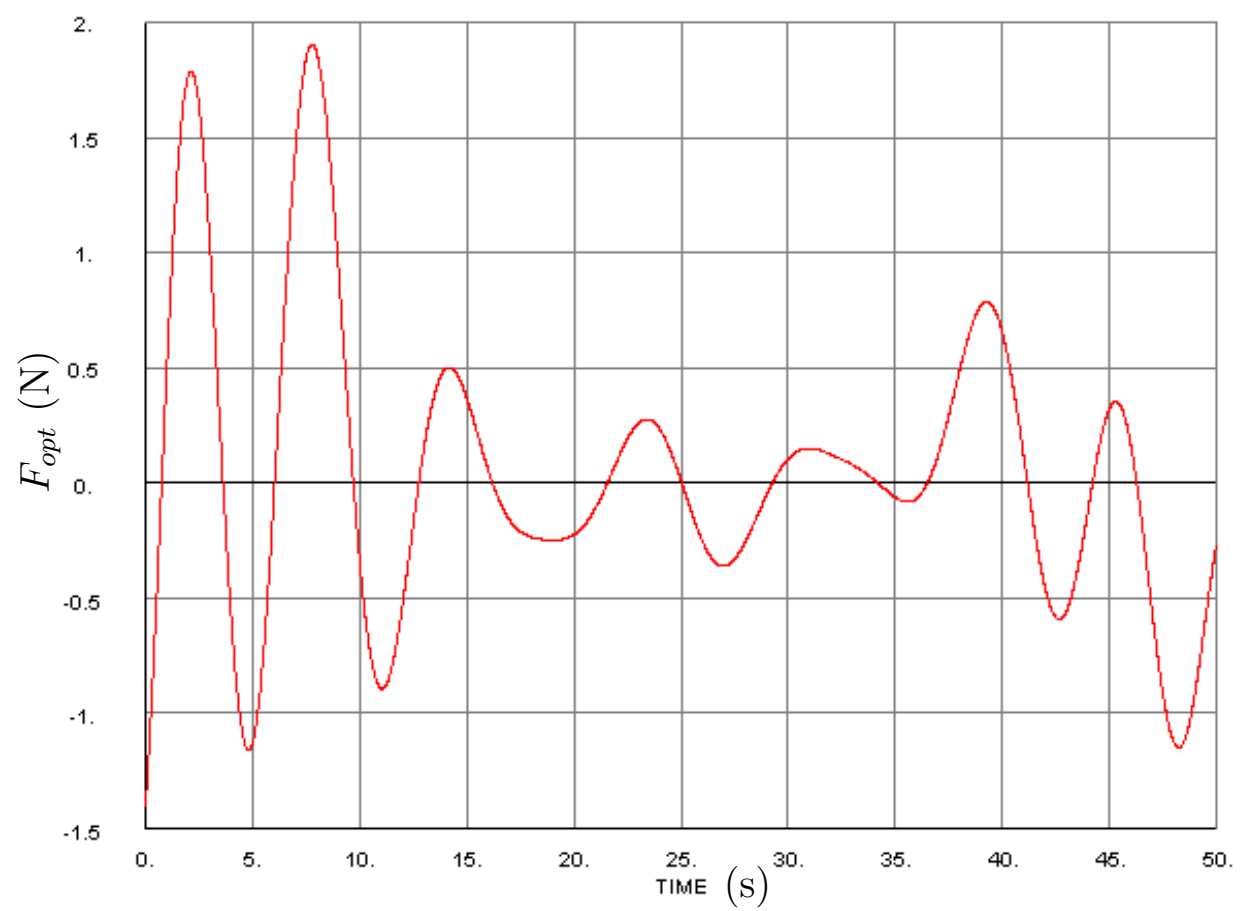

Figure 13: Optimal control 


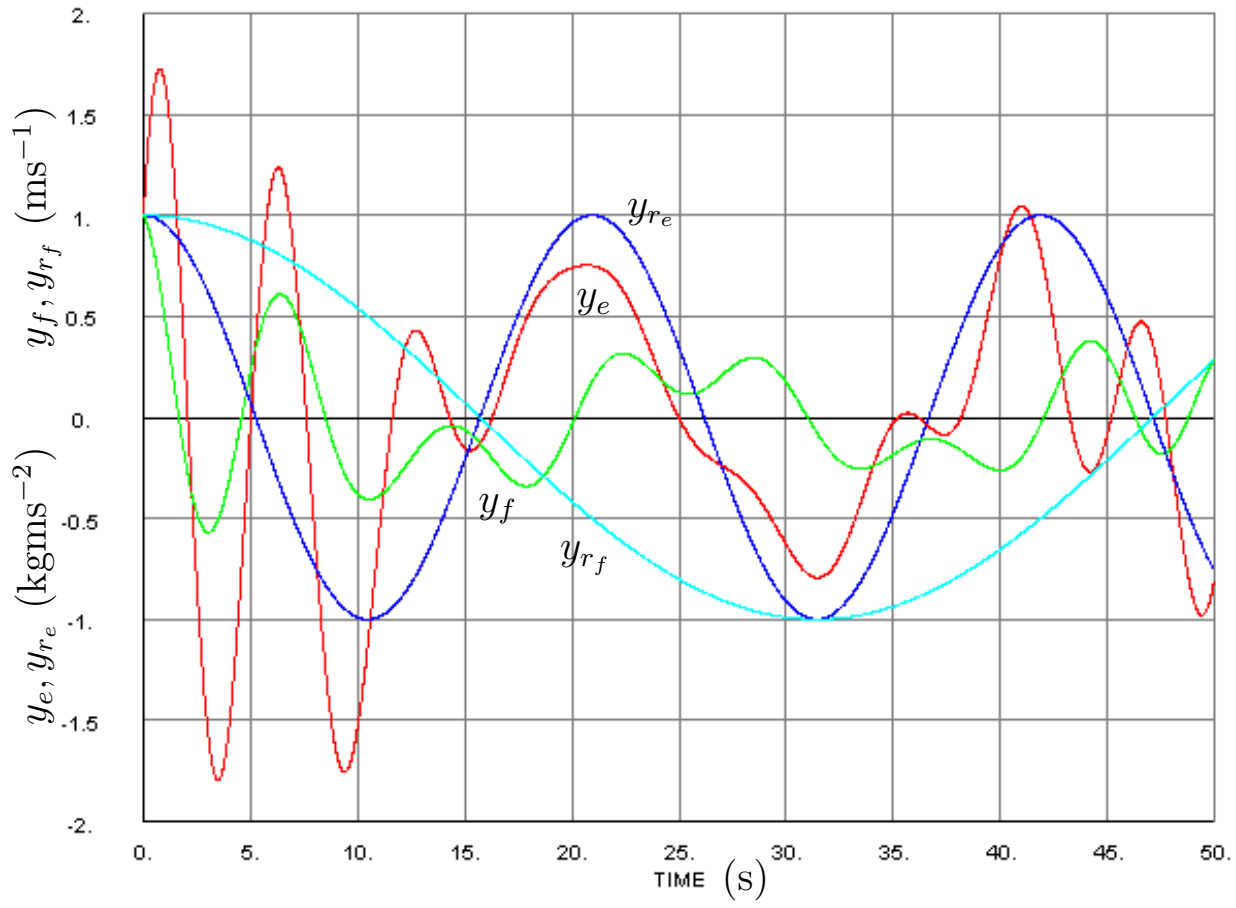

Figure 14: Outputs and their specified trajectories

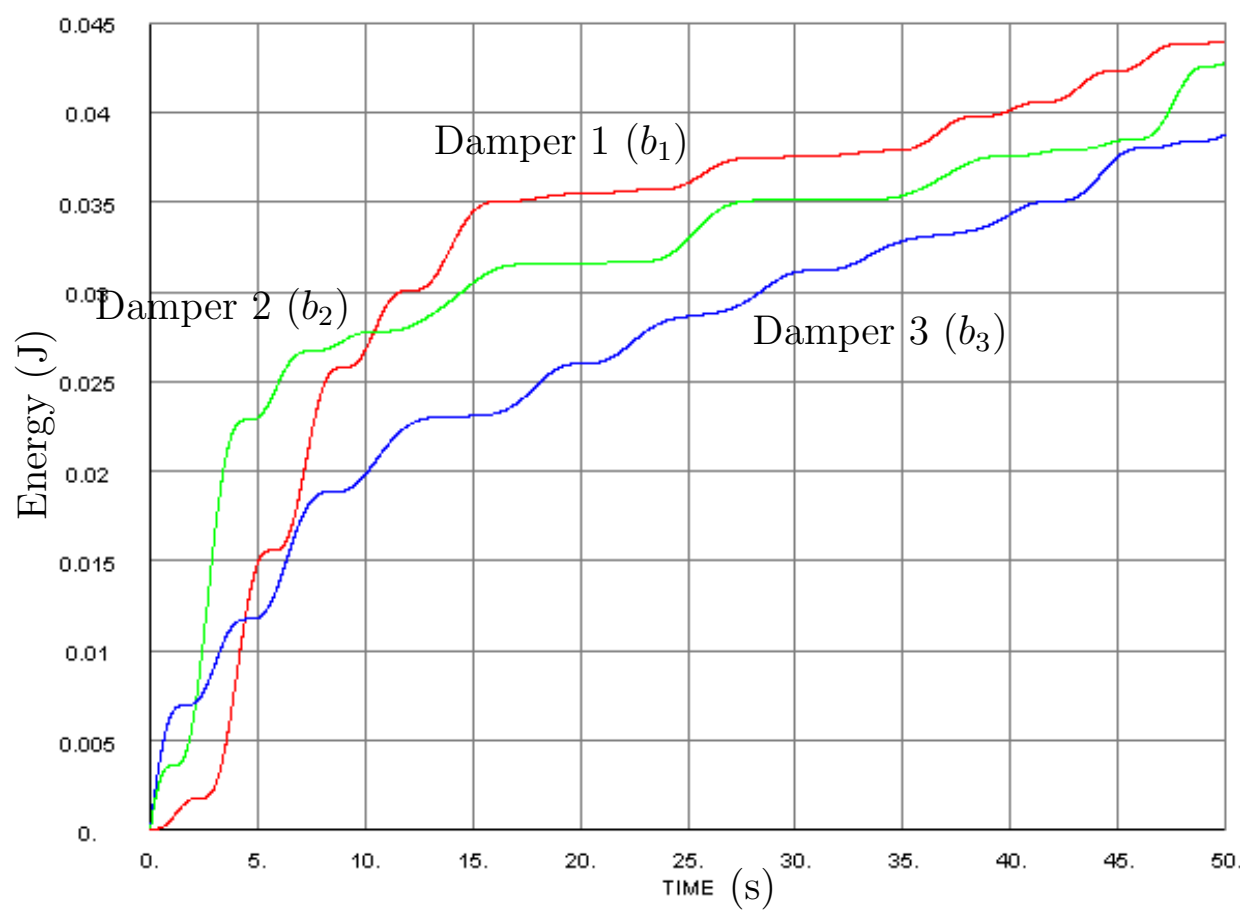

Figure 15: Dissipated energy in the dampers 
the potential for a systematic construction of bond graph that represents the optimal control formulation and the model for which the control is designed.

As shown in this paper, introducing optimisation into bond graph language brings more insight and a new vision about optimisation. Moreover coupling optimisation and bond graph use have System Engineering arguments. In fact a perspective is to extend the methodology of mechatronic system sizing on dynamic and energy criteria. With this in view; the integration of actuating line component specifications, optimal control, multivariable control, energy minimization in a context of sustainable development, design specifications not precisely defined and structure synthesis can all be dealt with using this procedure. Optimal control is the first step with the perspective of introducing a more general optimisation problem into bond graph language.

\section{References}

[1] Breedveld, P. C.: Multibond graph elements in physical systems theory, The Journal of the Franklin Institute, vol. 319(1/2), pp. 1-36, 1985.

[2] Breedveld, P. C.: A definition of the multibond graph language, in "Complex and Distributed Systems: Analysis, Simulation and Control", Tzafestas, S. and Borne, P., eds., Vol. 4 of "IMACS Transactions on Scientific Computing", pp. 69-72, North-Holland Publ. Comp., Amsterdam, 1986.

[3] Chereji B., O. Mouhib, W. Marquis-Favre, D. Thomasset, J. Pousin, M. Picq :An optimal control problem: bond graph representation and solver implementation, 5th MATHMOD, 8 - 10 February 2006, Vienna.

[4] Dauphin-Tanguy, G.: Les Bond Graphs. Herms Science Publications, Paris, 2000.

[5] Fotsu-Ngwompo, R.: Contribution au Dimensionnement des Systèmes sur des Critères Dynamiques et Energétiques - Approche par Bond Graph. Ph. D. Thesis: Institut National des Sciences Appliquées de Lyon, 1997.

[6] Fotsu-Ngwompo, R. and S. Scavarda: Dimensioning Problems in System Design Using Bicausal Bond Graphs. Simulation Practice and theory, vol. 7, 1999, pp. 577-587.

[7] Fotsu-Ngwompo, R., S. Scavarda, Thomasset, D., "Physical Model-Based Inversion in Control Systems Design Using Bond Graph Representation. Part 1: Theory." Proceedings of the IMECHE Part I Journal of Systems and Control Engineering, 2001, 215: 95-103.

[8] Fotsu-Ngwompo, R., S. Scavarda, Thomasset, D., "Physical Model-Based Inversion in Control Systems Design Using Bond Graph Representation. Part 2: Applications." Proc. of the IMECHE Part I Journal of Systems and Control Engineering, 2001, 215: 105-112.

[9] Gawthrop, P. J.: Bicausal Bond Graphs. In: Proc. of the ICBGM'95, 2nd Int. Conf. on Bond Graph Modeling and Simulation, Las Vegas, UsA, January, 1995, pp. 83-88.

[10] Gawthrop, P. J.: Physical Interpretation of Inverse Dynamics Using Bicausal Bond Graphs. Journal of the Franklin Institute 337 (2000), pp.743-769. 
[11] Groetsch, C. W., 1999. Inverse Problems. The Mathematical Association of America, UsA.

[12] Kailath, T., 1980. Linear Systems. Prentice Hall, Englewood Cliffs, NJ.

[13] Karnopp, D.C., Margolis, D.L., Rosenberg, R.C.: System Dynamics : Modeling and Simulation of Mechatronic Systems. John Wiley \& Sons, New York, 2000.

[14] Kirk D. : Optimal Control Theory, Prentice-Hall, 1970

[15] Marquis-Favre, W.; B. Chereji; D. Thomasset; S. Scavarda.: Bond Graph Representation of an Optimal Control Problem: the DC Motor Example. In: Proc. of the 7th ICBGM, 2005, New-Orleans, UsA.

[16] Maschke, B., van der Schaft, A. J.: Port-controlled Hamiltonian systems: Modelling origins and system-theoretic properties, in Proc. 2nd IFAC NOLCOS, Bordeaux, pp. 282-288, 1992.

[17] Mouhib O., B. Chereji, W. Marquis-Favre, D. Thomasset, J. Pousin, M. Picq: Procedure for the bond graph construction of an optimal control problem, In: International Journal of Tomography \& Statistics. Special Issue on: Control Applications of Optimisation - control and aeronautics, optimal control, control of partial differential equations. Vol. 5, Winter 2007

[18] Mouhib O., B. Chereji, W. Marquis-Favre, E. Bideaux D. Thomasset: Bond graph representation of an optimal control problem for output error minimization, 8th ICBGM 15 - 17 January 2007, San Diego CA.

[19] van der Schaft, A. J., Maschke, B.: Hamiltonian formulation of bond graphs, Ecole d't d'Automatique de Grenoble, Session 22, Brogliato, B., 17-21 sept 2001.

[20] Zadeh, L. A., Desoer, C. A., 1963. Linear System Theory. McGraw Hill Book Company, New York.

[21] Agrawal, S. K. and Fabien, B. C. Optimization of dynamic systems, volume 70 of Solid Mechanics and its Applications. Kluwer Academic Publishers, Dordrecht, Boston, London, 1999.

[22] Dolcini, P., Canudas de Wit, C., Bchart, H. Improved optimal control of dry clutch engagement. In $16^{\text {th }}$ IFAC World Congress, page 6, Prague, Czech Republic, 4-8 July 2005.

[23] Foulard, C., Gentil, S., Sandraz, J.-P. Commande et rgulation par calculateur numrique. Editions Eyrolles, 1979.

[24] Moler, C., van Loan, C. Nineteen dubious ways to compute the exponential of a matrix, twenty-five years later. Society for Industrial and Applied Mathematics Review, 45(1):1-45, 2003.

[25] MS1. http://www.lorsim.be, 2004.

\section{Appendix}




\section{A Partial dualization of multiport R-elements}

The generic bond graph representation used for the proof of the procedure proposed is based on the dualization concept [1]. Dualization is realized by a symplectic gyrator or unit gyrator (Fig. 16-a) and enables the nature of the power variables to be exchanged. The implicit constitutive relation of this element is given by :

$$
\left(\begin{array}{l}
e_{1} \\
e_{2}
\end{array}\right)-\left(\begin{array}{ll}
0 & I \\
I & 0
\end{array}\right) \cdot\left(\begin{array}{l}
f_{1} \\
f_{2}
\end{array}\right)=0
$$

One important property is that two symplectic multiport gyrators in series are equivalent to a multibond (Fig. 16-b). In the context of this paper, this multiport element has been used to simplify the development of the proof of the procedure effectiveness by means of partial dualization presented hereafter.

$$
\begin{aligned}
& \text { (a) } \frac{e_{1}}{f_{1}} \underset{\left(\begin{array}{ll}
0 & I \\
I & 0
\end{array}\right)^{\frac{e_{2}}{f_{2}}}}{\mathbb{S}} \\
& \text { (b) } \quad\left(\begin{array}{ll}
0 & I \\
I & 0
\end{array}\right) \|\left(\begin{array}{ll}
0 & I \\
I & 0
\end{array}\right)
\end{aligned}
$$

Figure 16: Symplectic gyrator: (a) representation, (b) one property

Concerning R-elements, this dualization enables us to deal with a unique type of causality (namely resistance causality). Consider the figure 17-a causal multiport R-element collecting all individual 1-port or multiport R-elements of a general causal bond graph. Without loss of generality the ports of this multiport R-element have been classified in such a way that the first ones correspond to the resistance causality while and the last ones to the conductance causality. The use of a multiport symplectic gyrator enables the conductance causality ports to be dualized (Fig. 17-b). The equivalence between both R-element constitutive matrices is shown by :

$$
\begin{gathered}
\left\{\begin{array}{l}
e_{\mathrm{r}}-R_{\mathrm{rr}} \cdot f_{\mathrm{r}}-R_{\mathrm{rc}} \cdot f_{\mathrm{c}}=0 \\
e_{\mathrm{c}}-R_{\mathrm{cr}} \cdot f_{\mathrm{r}}-R_{\mathrm{cc}} \cdot f_{\mathrm{c}}=0
\end{array}\right. \\
\Leftrightarrow\left\{\begin{array}{l}
e_{\mathrm{r}}=\left(R_{\mathrm{rr}}-R_{\mathrm{rc}} R_{\mathrm{cc}}^{-1} R_{\mathrm{cr}}\right) \cdot f_{\mathrm{r}}+R_{\mathrm{rc}} R_{\mathrm{cc}}^{-1} \cdot e_{\mathrm{c}} \\
f_{\mathrm{c}}=-R_{\mathrm{cc}}^{-1} R_{\mathrm{cr}} \cdot f_{\mathrm{r}}+R_{\mathrm{cc}}^{-1} \cdot e_{\mathrm{c}}
\end{array}\right. \\
\Leftrightarrow e_{\mathrm{R}}=R_{\mathrm{R}} \cdot f_{\mathrm{R}}
\end{gathered}
$$

with $e_{\mathrm{R}}=\left(\begin{array}{c}e_{\mathrm{r}} \\ f_{\mathrm{c}}\end{array}\right)$ and $f_{\mathrm{R}}=\left(\begin{array}{c}f_{\mathrm{r}} \\ e_{\mathrm{c}}\end{array}\right)$. 


$$
\begin{aligned}
& \begin{array}{l}
e_{\mathrm{r}} \\
\hline f_{\mathrm{r}}
\end{array} \\
& \text { (a) } \\
& \frac{e_{\mathrm{c}}}{f_{\mathrm{c}}} \mathbb{R}: R=\left[\begin{array}{ll}
R_{\mathrm{rr}} & R_{\mathrm{rc}} \\
R_{\mathrm{cr}} & R_{\mathrm{cc}}
\end{array}\right] \\
& \text { III } \\
& \text { (b) } \quad \frac{e_{\mathrm{r}}}{f_{\mathrm{r}}} \\
& \underset{f_{\mathrm{c}}}{e_{\mathrm{c}}} \mathbb{S G Y} \mid \begin{array}{ll}
f_{\mathrm{r}} \\
\frac{e_{\mathrm{c}}}{\mathrm{c}}
\end{array} \mathbb{R}: R_{\mathrm{R}}=\left[\begin{array}{cc}
R_{\mathrm{rr}}-R_{\mathrm{rc}} R_{\mathrm{cc}}^{-1} R_{\mathrm{cr}} & R_{\mathrm{rc}} R_{\mathrm{cc}}^{-1} \\
-R_{\mathrm{cc}}^{-1} R_{\mathrm{cr}} & R_{\mathrm{cc}}^{-1}
\end{array}\right]
\end{aligned}
$$

Figure 17: Partial dualization of multiport R-elements: (a) original representation, (b) dualized representation

Correspondence between $R_{\mathrm{R}}^{\mathrm{T}}$ and $T$ The proof of the procedure's effectiveness shows the appearance of the matrix $R_{\mathrm{R}}^{\mathrm{T}}$ in the optimizing bond graph (Fig. 8). It is important to see the correspondence with the general bond graph representation (Fig. 2). Starting with the matrix $R_{\mathrm{R}}^{\mathrm{T}}$ (Fig. 18-a)the corresponding matrix $T$ is obtained by :

$$
\begin{gathered}
\left\{\begin{array}{l}
e_{\text {opt.r }}=\left(R_{\mathrm{rr}}-R_{\mathrm{rc}} R_{\mathrm{cc}}^{-1} R_{\mathrm{cr}}\right)^{\mathrm{T}} \cdot f_{\text {opt.r }}-\left(R_{\mathrm{cc}}^{-1} R_{\mathrm{cr}}\right)^{\mathrm{T}} \cdot e_{\mathrm{opt} . \mathrm{c}} \\
f_{\text {opt.c }}=\left(R_{\mathrm{rc}} R_{\mathrm{cc}}^{-1}\right)^{\mathrm{T}} \cdot f_{\text {opt.r }}+\left(R_{\mathrm{cc}}^{-1}\right)^{\mathrm{T}} \cdot e_{\text {opt.c }}
\end{array}\right. \\
\Leftrightarrow\left\{\begin{array}{l}
e_{\text {opt.r }}=R_{\mathrm{rr}}^{\mathrm{T}} \cdot f_{\text {opt.r }}-R_{\mathrm{cr}}^{\mathrm{T}} \cdot f_{\text {opt.c }} \\
e_{\text {opt.c }}=-R_{\mathrm{rc}}^{\mathrm{T}} \cdot f_{\text {opt.r }}+R_{\mathrm{cc}}^{\mathrm{T}} \cdot f_{\text {opt.c }}
\end{array}\right.
\end{gathered}
$$

One important feature of the matrix $T$ is that it is causality dependent.

Correspondence between $-R_{\mathrm{R}}^{\mathrm{T}}$ and $-R^{\mathrm{T}} \quad$ Based on a similar development as in the previous paragraph, it is not difficult to show that the matrix $-R_{\mathrm{R}}^{\mathrm{T}}$ in the figure 8 bond graph corresponds to the matrix $-R^{\mathrm{T}}$ in the figure 2 bond graph representation.

In the particular case where the R-elements are 1-port elements, the matrix $R$ is diagonal and $R^{\mathrm{T}}=T=R$ which simplifies the final matrices that are causality independant.

\section{B Bicausality}

The bicausality concept [9] is an extension of the causality concept which corresponds to a strict mathematical point of view on a power bond. Considering figure 19-a acausal bond graph representation of a power bond, this can be viewed as a graphical representation of a power connection between two subsystem power ports, thus forcing the power variables (effort and flow) to be identical. The mathematical representation of this power port connection can be 


$$
\begin{aligned}
& \text { (a) } \underset{f_{\text {opt.r }}}{f_{\text {opt.r }}} \mathbb{R}: R_{\mathrm{R}}^{\mathrm{T}}=\left[\begin{array}{cc}
\left(R_{\mathrm{rr}}-R_{\mathrm{rc}} R_{\mathrm{cc}}^{-1} R_{\mathrm{cr}}\right)^{\mathrm{T}} & -\left(R_{\mathrm{cc}}^{-1} R_{\mathrm{cr}}\right)^{\mathrm{T}} \\
\left(R_{\mathrm{rc}} R_{\mathrm{cc}}^{-1}\right)^{\mathrm{T}} & \left(R_{\mathrm{cc}}^{-1}\right)^{\mathrm{T}}
\end{array}\right] \\
& \text { III } \\
& \text { (b) } f_{\text {opt.r }}
\end{aligned}
$$

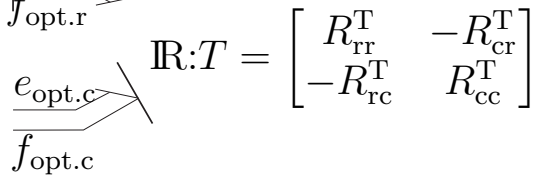

Figure 18: Bond graph correspondence between (a) $R_{\mathrm{R}}^{\mathrm{T}}$ and (b) $T$

expressed by the two implicit equations :

$$
\left\{\begin{array}{l}
e_{1}-e_{2}=0 \\
f_{1}-f_{2}=0
\end{array}\right.
$$

Causality corresponds to the organisation of these two equations with a strong physical interpretation and gives both the assignment possibilities of the figure 19-b causal power bonds. However inspection of the previous implicit equations show that from a strict mathematical point of view, it is possible to have two other calculus schemes displayed in the figure 19-c, these are called bicausal power bonds. This bicausal assignment has no physical interpretation. It only means that both power variables are mathematically determined at the same time by the same subsystem set of equations. The interest of using bicausality, and thus this assignment, becomes obvious for deriving inverse models $[6,7,10]$. In the bicausity assignment the stroke is split into two half-strokes, one dedicated to effort assignment (half-arrow opposite side), and the other one dedicated to flow assignment (half-arrow side).

It is now necessary to introduce two new elements in the representation that, on one side, initiate a bicausality assignment, and, on the other side, properly terminate this bicausal assignment. The element from which bicausality starts is a double source (see Fig. 20-a) and the element where bicausality terminates is a double sensor (see Fig. 20-b).

Concerning the bond graph bicausal assignment, the element constraints are the same as for the causality assignment. The difference resides in the fact that effort and flow assignments are now uncoupled. In the bicausal bond graph, bicausality and causality coexist but junction constraints show that two bicausal paths cannot be adjacent. Finally bicausality is assigned from a double source to a double sensor, this means that these elements are necessarly present in pairs. 


$$
\begin{gathered}
\text { (a) } \quad \frac{e_{1} \quad e_{2}}{f_{1} \quad f_{2}} \\
\text { (b) }\left\{\begin{array} { l } 
{ e _ { 1 } : = e _ { 2 } } \\
{ f _ { 2 } : = f _ { 1 } }
\end{array} \quad \left\{\begin{array}{l}
e_{2}:=e_{1} \\
f_{1}:=f_{2}
\end{array}\right.\right. \\
\left\{\begin{array} { l } 
{ e _ { 1 } : = e _ { 2 } } \\
{ f _ { 1 } : = f _ { 2 } }
\end{array} \quad \left\{\begin{array}{l}
e_{2}:=e_{1} \\
f_{2}:=f_{1}
\end{array}\right.\right.
\end{gathered}
$$

Figure 19: Power bond (a) acausal, (b) in causal assignment, and (c) in bicausal assignment

$$
\begin{aligned}
& \text { (a) } \begin{array}{ll}
E, F & \\
\text { SëSf } & e
\end{array} \quad \begin{array}{l}
e:=E \\
f:=F
\end{array} \\
& \text { (b) } \begin{array}{lll}
u, y \uparrow & e & u:=e \\
\operatorname{DeDf} L & y & :=f
\end{array}
\end{aligned}
$$

Figure 20: (a) Double source, (b) double sensor 